\title{
Spinal Cord Stimulation as a Treatment Option for Intractable Neuropathic Cancer Pain
}

\author{
Alexander E. Yakovlev, MD and Yakub Ellias, MD
}

\begin{abstract}
Nearly 6,750,000 people suffer moderate to severe cancer-related pain each year. Unfortunately, $10 \%$ to $15 \%$ of these patients fail to achieve acceptable pain relief with conventional management. Spinal cord stimulation (SCS) has been used with increased frequency for successful treatment of intractable cancer pain. We present two cases of intractable, refractoryto-conventional treatment cancer pain that were successfully treated with SCS. Case I reports a $5 \mathrm{I}$-year-old male with burning pain at the left groin site of inguinal metastases, post-surgical and intraoperative radiation therapy for treatment of squamous cell carcinoma of the anus. Case 2 reports a 43-year-old woman with intractable, burning, throbbing, and shooting pain, post-debulking followed by radiation of a metastatic colon carcinoma. In both cases SCS implantation provided $90 \%$ to $100 \%$ pain relief, improved functioning and sleep, and discontinuation of pain medications, sustained through 12 months.
\end{abstract}

Keywords: Cancer pain; Neuropathic pain; Post irradiation neuropathy; Spinal cord stimulation

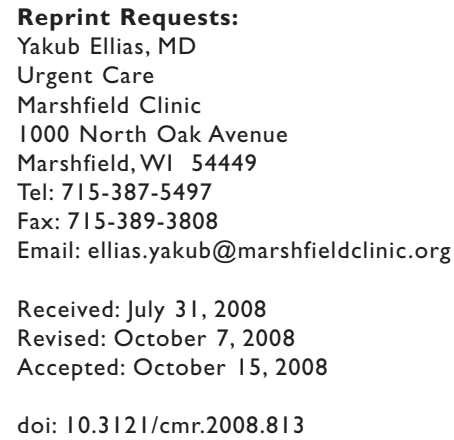

\section{Case Presentations}

Case 1

The patient is a 51-year-old male who underwent anterior-posterior resection and intraoperative radiation therapy for squamous cell carcinoma of the anus. He later developed inguinal adenopathy, which was also irradiated because it contained secondary metastasis from the carcinoma of the anus. Approximately 4 months post-irradiation, he developed burning pain in the left groin at the site of the inguinal metastasis. The pain was described as hot and burning, occasionally sharp and shooting, with visual analog scale (VAS) scores at rest of 2 to 4 out of 10 in severity. The patient was most aggravated by the fact that, during activity, the pain escalated to a VAS score of 8 out of 10 and significantly limited his activities. Occasionally, sitting or getting off his feet would afford him some comfort; however, the pain never disappeared entirely.

The patient had used ibuprofen, acetaminophen, gabapentin, cyclobenzaprine, amitriptyline, and topical ointments, but they did not provide any relief. Because codeine, oxycodone, and hydrocodone had caused a rash in the past, the use of opioids was limited. Darvocet, fentanyl patches, and ilioinguinal and iliohypogastric nerve blocks provided the patient with minimal pain relief. $\mathrm{He}$ was counseled on alternative options of treatment, including an intrathecal drug delivery system and spinal cord stimulation (SCS). He chose SCS.

Once the decision has been made to proceed to SCS, a successful trial of percutaneous placement of temporary epidural leads must be performed before implantation of the entire SCS system. These temporary leads are connected to an external pulse generator, and patients typically wear them for a few days while 


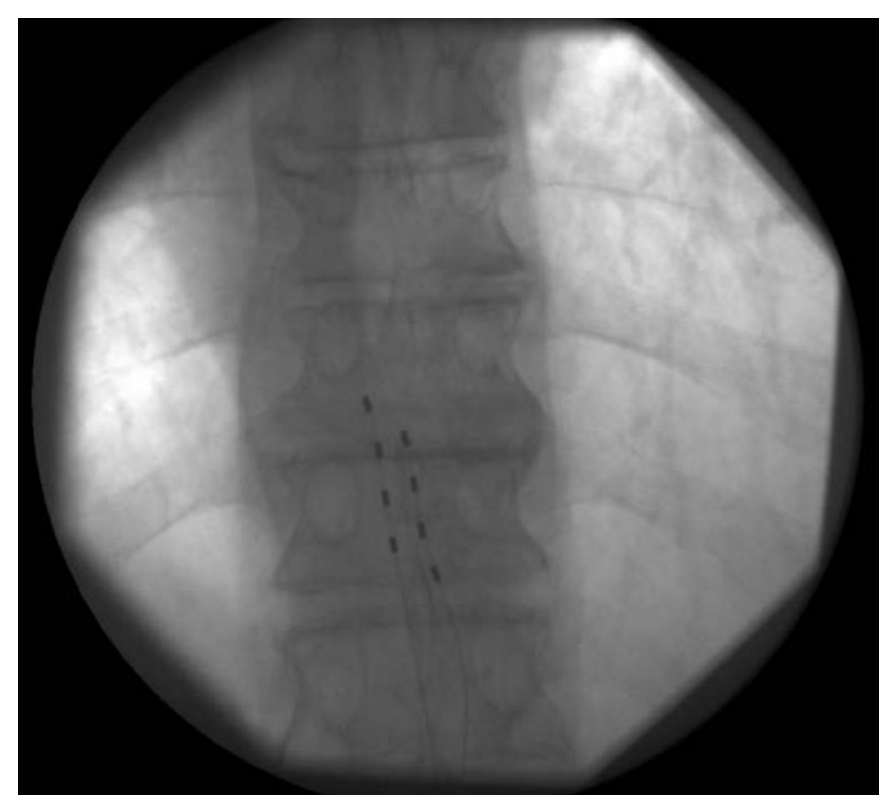

Figure 1. Thoracic epidural placement of two quadripolar leads showing the electrodes in a staggered position with tips at T-8.

performing their usual activities of daily living (ADLs) to fully assess the efficacy of the SCS trial. A realistic assessment presumes the enhancement of ADLs and decreased pain levels by $50 \%$ or more.

The surgical technique for final implantation of the SCS system is quite simple, requiring approximately 1 hour to perform the procedure in an ambulatory surgery setting. Like any surgical patient, patients who undergo SCS implantation are at risk for the standard surgical complications including bleeding, infection, cerebrospinal fluid leak, and hardware failure; however, these events are quite rare in experienced centers.

The patient successfully underwent a trial of percutaneous placement of two quadripolar epidural leads (Medtronic Inc, Minneapolis, MN) to level T8-T9 (figure 1). During the SCS trial, he reported good pain control (VAS score of 1 to 2 out of 10). Two weeks later, the patient underwent implantation with permanent leads and a Synergy generator (Medtronic Inc.). The procedure was done in ambulatory surgery, and his postoperative course was uneventful. The set stimulation parameters were amplitude of 3 to 6 volts, pulse width of 210 microseconds, and frequency of $100 \mathrm{~Hz}$. The patient used the spinal cord stimulator continuously and reported $100 \%$ pain relief both at rest and with activities. He stopped using all pain medications and returned to his pre-neuropathy level of functioning. At 12-months post-implant, the patient continued to report good pain control (VAS scores 1 to 2 out of 10) and improved functional status.

\section{Case 2}

The patient is a 43-year-old woman who presented to the pain clinic with complaints of intractable, burning, throbbing, and shooting pain in her low back and right lower extremity that developed 3 months after debulking of a metastatic epidural tumor in the low thoracic spine from carcinoma of the colon followed by radiation therapy. The patient was not found to have recurrent metastatic disease. The pain was considered to be radiation induced. Reported VAS scores ranged from 5 to 9 out of 10 . The patient tried various non-opioid and opioid analgesics, including ibuprofen, acetaminophen, codeine, oxycodone, hydrocodone, morphine, fentanyl patches, gabapentin, topiramate, tizanidine, clonidine patch, amitriptyline and topical ointments, all of which failed to provide significant pain relief. Moreover, a series of caudal epidural steroid blocks did not alleviate the severe neuropathic pain.

The patient was offered additional treatment options, including an intrathecal drug delivery system and spinal cord stimulator. She declined intrathecal pump placement and instead chose to proceed with placement of a spinal cord stimulator which she thought was a less invasive therapy. A trial of percutaneous placement as previously described for case 1 was undertaken prior to final placement of the SCS system. She successfully underwent a trial of percutaneous placement of two epidural quadripolar leads (Medtronic Inc) to level T9-T10 (figure 2). During the SCS trial, she reported adequate pain control with a VAS score of 1 out of 10 . Three weeks later, she was implanted with permanent leads and a Synergy generator (Medtronic Inc). The procedure was done in ambulatory surgery, and her postoperative course was uneventful. The set stimulation parameters were amplitude of 2 to 7 volts, pulse width of 300 microseconds, and frequency of $60 \mathrm{~Hz}$. The patient used the spinal cord stimulator continuously. After the surgery, she reported $90 \%$ to $100 \%$ pain relief, and she stopped using opioids. She reported an

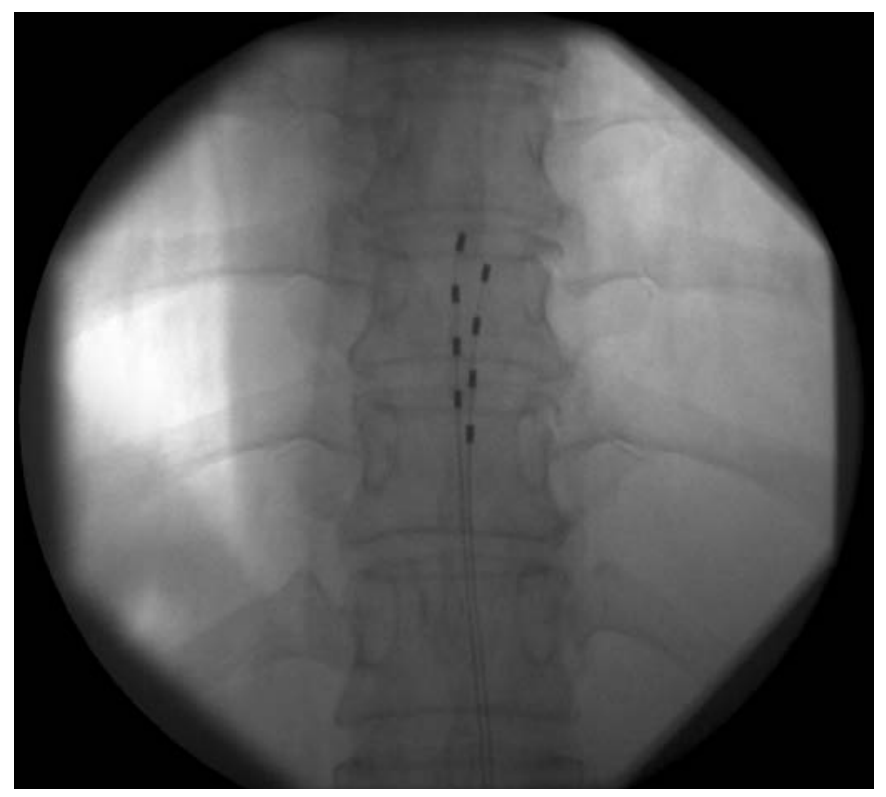

Figure 2. Thoracic epidural placement of two quadripolar leads showing the electrodes in a staggered position with tips at T-9. 
increased level of functioning and improved sleep. In the 12 months post-implant, the patient has reported sustained pain relief and continued improvements in functioning.

\section{Discussion}

Approximately 9 million people suffer from cancer-related pain each year, and nearly $75 \%$ of these people experience moderate-to-severe pain in the advanced and terminal stages of their disease. ${ }^{1}$ The World Health Organization (WHO) guidelines for cancer pain management are based on a three-step ladder: (1) non-steroidal anti-inflammatory drugs (NSAIDs), aspirin, and acetaminophen for mild-to-moderate levels of cancer pain; (2) weak opioids for mild-to-moderate pain that does not respond to NSAIDs alone; and (3) strong opioids for moderate-to-severe levels of cancer pain. ${ }^{2}$ Adjuvant medications, such as antiepileptics and tricyclic antidepressants, can also be added at any step of the ladder for optimal pain relief.

Unfortunately, approximately $10 \%$ to $15 \%$ of patients with cancer-related pain do not achieve acceptable levels of pain relief with opiates alone or in combination with conventional adjuvant analgesics. ${ }^{3}$ Unconventional agents and interventional management approaches have received considerable attention in an attempt to provide increased pain relief for patients with intractable cancer pain. SCS has been used with increased frequency for the treatment of intractable cancer pain.

\section{SCS as an Analgesic}

SCS was first used for pain control in 1967 by neurosurgeon Dr. Norman Shealy and his colleagues. ${ }^{4}$ SCS is based on the principles enunciated in the "gate-control theory" of pain proposed by Melzack and Wall in 1965.5 This theory postulates that SCS activates large-diameter afferent fibers via application of an externally applied electric field that "closes the gate" to pain transmission. SCS blocks pain by stimulating the dorsal columns, which inhibits transmission through the pain-conducting spinothalamic tract. The electrical pulse wave is generated either with an external neuropulse generator that transmits the electrical pulse via a cable to an antenna worn externally which is radiocoupled to an implanted receiving device or with an implanted, programmable neuropulse generator. The newly developed programmable generators contain an antenna, computer module, and a non-rechargeable or rechargeable battery pack which can last up to 9 years. By placing an electrode array to different segments of the spinal cord, the patient's subjective pain can be controlled in nearly all areas of the body, from C1-C2 for facial pain, to $\mathrm{T} 7$ for abdominal pain, or to $\mathrm{T} 8 \mathrm{-T} 9$ for low back and radicular pain.

Since its first use over 3 decades ago, in which electrodes were placed epidurally over the dorsal columns of the spinal cord, SCS has been further refined, and multiple studies have demonstrated its efficacy in the treatment of intractable, chronic pain with a variety of causes. ${ }^{6}$ SCS has been used to successfully treat chronic pain in patients with failed back syndrome, ${ }^{7,8}$ ischemic limb pain, ${ }^{9}$ angina pectoris, ${ }^{10}$ and painful peripheral neuropathies. ${ }^{11,12}$

\section{SCS for Cancer Pain}

It has been estimated that approximately $15 \%$ to $40 \%$ of chronic cancer pain has a neuropathic component, ${ }^{13}$ and neuropathic pain often responds poorly to opioids. Moreover, the side effect profile of opioids and other analgesics often warrant other interventional pain management approaches. SCS for cancer pain has been receiving recent attention as a safe and efficacious treatment option.

There are other reports about the pain-alleviating effect of SCS for patients with cancer pain. Cata et al ${ }^{14}$ reported the benefits of SCS for chemotherapy-induced pain in two patients whose pain had been poorly controlled with conventional medications. Both patients reported pain relief of more than 50\%, improved gait, and decreased daily requirements of pain medications after SCS. Similarly, Hamid and Haider ${ }^{15}$ reported on the adequate pain control achieved with SCS in a patient with intractable neuropathic pain secondary to radiation-induced transverse myelitis unresponsive to other pain management interventions.

\section{Conclusion}

We present two cases of intractable, refractory-toconventional treatment cancer pain which were successfully treated with SCS. This technique may be a therapeutic alternative for patients who have exhausted all available treatments or who have an increased risk for or prefer not to have more invasive interventions. There are no risks for potential neurologic dysfunctions which can complicate ablative procedures. With this method of treatment, our patients had satisfactory symptom relief, an increase in ADLs and discontinuation of pain medications. In our opinion, SCS is a relatively easy to perform, effective, and safe procedure and is reversible should patients lose its pain-alleviating effect. Moreover, if SCS fails to provide the expected level of pain relief, patients are not required to undergo the uncomfortable weaning process associated with intrathecal or epidural pain medications.

SCS is an important adjuvant treatment in patients with intractable neuropathic cancer pain, which may make its own niche in the therapy algorithm for this group of patients. These two cases presented here represent additional evidence and support for utilizing SCS in the treatment of refractory neuropathic cancer pain.

\section{Acknowledgments}

The authors thank Marshfield Clinic Research Foundation for its support through the assistance of Linda Weis, Alice Stargardt and Jennifer Hayes in the preparation of this article. 


\section{References}

1. Gralow I. Cancer pain: an update of pharmacological approaches in pain therapy. Current Opinion in Anaesthesiology 2002;15(5):555-561.

2. Schug SA, Zech D, Dorr U. Cancer pain management according to WHO analgesic guidelines. J Pain Symptom Manage 1990;5:27-32.

3. Sloan PA, Melzack R. Long-term patterns of morphine dosage and pain intensity among cancer patients. Hosp J 1999; 14:35-47.

4. Shealy CN. Dorsal column stimulation. Surg Neurol 1977;7:192.

5. Melzack RA, Wall PD. Pain mechanisms: a new theory. Science 1965;150:971-979.

6. Cameron T. Safety and efficacy of spinal cord stimulation for the treatment of chronic pain: a 20-year literature review. J Neurosurg 2004;100:254-267.

7. Struijk JJ, Holsheimer J, Spincemaille GH, Gielen FL, Hoekema $\mathrm{R}$. Theoretical performance and clinical evaluation of transverse tripolar spinal cord stimulation. IEEE Transactions in Rehabilitation Engineering 1998:6:277-285.

8. Ohnmeiss DD, Rashbaum RF, Bogdanorffy GM. Prospective outcome evaluation of spinal cord stimulation in patients with intractable leg pain. Spine 1996;21:1344-1350.

9. Ghajar AW, Miles JB. The differential effect of the level of spinal cord stimulation on patients with advanced peripheral vascular disease in the lower limbs. Br J Neurosurg 1996;12:402-408.

10. Hautvast RW, DeJongste MJ, Staal MJ, van Gilst WH, Lie KI. Spinal cord stimulation in chronic intractable angina pectoris: a randomized controlled efficacy study. Am Heart J 1998;136:1114-1120.

11. Kumar K, Toth C, Nath RK. Spinal cord stimulation for chronic pain in peripheral neuropathy. Surg Neurol 1996;46:363-369.

12. Tesfaye S, Watt J, Benbow SJ, Pang KA, Miles J, McFarlane IA Electrical spinal-cord stimulation for painful diabetic peripheral neuropathy. Lancet 1996;348:1698-1701.

13. Berger A, Dukes E, Mercadante S, Oster G. Use of antiepileptics and tricyclic antidepressants in cancer patients with neuropathic pain. Eur J Cancer Care 2005;15:138-145.

14. Cata JP, Cordella JV, Burton AW, Hassenbusch SJ, Weng H, Dougherty PM. Spinal cord stimulation relieves chemotherapy-induced pain: a clinical case report. J Pain Symptom Manage 2004;27:72-78.

15. Hamid B, Haider N. Spinal cord stimulator relieves neuropathic pain in a patient with radiation-induced transverse myelitis. Pain Pract 2007;7:345-347.

\section{Author Affiliations}

Yakub Ellias, $M D$

Marshfield Clinic

Department of Urgent Care

Marshfield, Wisconsin

Alexander E. Yakovlev, MD

Marshfield Clinic

Department of Interventional Pain Management

Marshfield, Wisconsin

Present Address:

Comprehensive Pain Management of the Fox Valley, SC

820 East Grant Street, Suite 335

Appleton, Wisconsin 54911 